\title{
Determinación de los parámetros de diseño, manufactura y producción de sellos en plástico.
}

\section{Determination of parameters for design, manufacture and production of plastisol stamps}

Miguel Ángel Escobar Guachambala. ${ }^{1}$, Sócrates Miguel Aquino Arroba. ${ }^{2}$, Edwin Rodolfo Pozo Safla. ${ }^{3} \&$ Luis Santiago Choto Chariguaman. ${ }^{4}$

Recibido: 19-04-2019 / Revisado: 25-05-2019 /Aceptado: 24-06-2019/ Publicado: 15-07-2019

In this research, the different stages for production of stamps in plastisol. In the first stage, the stamp modeling are developed and it is corresponding mold are generated by computer-aided design software. In the second stage, the machining methods used to manufacture the details of aforementioned stamp were determined; The partial machining of cavities is done by computer numerical control and the finishing is developed by the process of electroerosion by penetration, being necessary to manufacture several electrodes in the graphite by computer-aided manufacturing software.

In the third stage, the mold for the production of stamps was obtained, the plastisol was deposited with dyes in the cavities by means of a micro-injection process, then the curing was carried out by means of heat at $300{ }^{\circ} \mathrm{C}$. In the production stage the proportions of mixture of resin with the dyes, curing temperature, curing times and cooling are produced. Finally, we obtained a product of good quality according to the

1 Escuela Superior Politécnica de Chimborazo, Facultad de Mecánica. Riobamba, Ecuador. maescobar@espoch.edu.ec

2 Escuela Superior Politécnica de Chimborazo, Facultad de Mecánica. Riobamba, Ecuador. saquino@espoch.edu.ec

3 Escuela Superior Politécnica de Chimborazo, Facultad de Mecánica. Riobamba, Ecuador. edwin.pozo@espoch.edu.ec

4 Escuela Superior Politécnica de Chimborazo, Facultad de Mecánica. Riobamba, Ecuador. lchoto@espoch.edu.ec 
design specifications, getting the stated objective. It is recommended to automate the process of micro injection of plastisol in the cavities of the mold, to reduce the production time.

Keywords: CAD CAM, Mold, Plastisol

\section{Resumen.}

En esta investigación, se desarrolló las diferentes etapas para la producción del sello en plastisol. En la primera etapa se desarrolló el modelado del sello y su correspondiente molde mediante software de diseño asistido por computador. En la segunda etapa, se determinó los métodos de mecanizado que se emplean para lograr mecanizar los detalles que tiene el sello anteriormente mencionado; el mecanizado parcial de las cavidades se lo realizó mediante mecanizado por control numérico computacional y el acabado de las mismas se realizó mediante un proceso de electroerosión por penetración, siendo necesario mecanizar varios electrodos en grafito mediante software de manufactura asistida por computador.

En la tercera etapa, se obtuvo el molde para la producción de los sellos, depositando el plastisol con colorantes en las cavidades mediante un proceso de micro-inyección, posteriormente se realizó el curado mediante calor a $300^{\circ} \mathrm{C}$.

En la etapa de producción se consideró las proporciones de mezcla de la resina con los colorantes, temperatura de curado, los tiempos de curado y enfriamiento. Finalmente se obtuvo un producto de buena calidad según las especificaciones del diseño, cumpliéndose el objetivo planteado. Se recomienda automatizar el proceso de micro inyección del plastisol en las cavidades del molde, para reducir el tiempo de producción.

Palabras claves: CAD CAM, molde, plastisol

\section{Introducción.}

En la actualidad los productos moldeados están presentes en cada equipo, máquina, vestimenta, accesorios, en otros. Los productos de uso común y demanda son los llaveros y accesorios en plastisol, por tener un costo de producción reducido. Para la obtención de un producto moldeado, se hace necesario utilizar tecnologías CAD CAE CAM, además del manejo de maquinaria $\mathrm{CNC}$ y de tener conocimiento de los procesos de manufactura relacionados. [1]

El diseño del producto se lo realiza de una manera planificada, de tal manera que los tiempos de mecanizado se reduzcan y que se lo pueda realizar con facilidad. El proceso de mecanizado 
mediante $\mathrm{CNC}$ es uno de los métodos de manufactura más precisos, para obtener partes: industriales, automotrices, entre otros.[2] Este proceso nos brinda la facilidad de producir productos con formas complejas y de forma eficiente. En la figura 1, se muestra una fresadora CNC con cuarto eje montable sobre la mesa de trabajo, gracias a la utilización de este tipo de maquinaria es posible en la actualidad obtener moldes para productos en plastisol con formas complejas con alta precisión. [3]

Figura. 1 Fresadora CNC ROMI D600 4x.

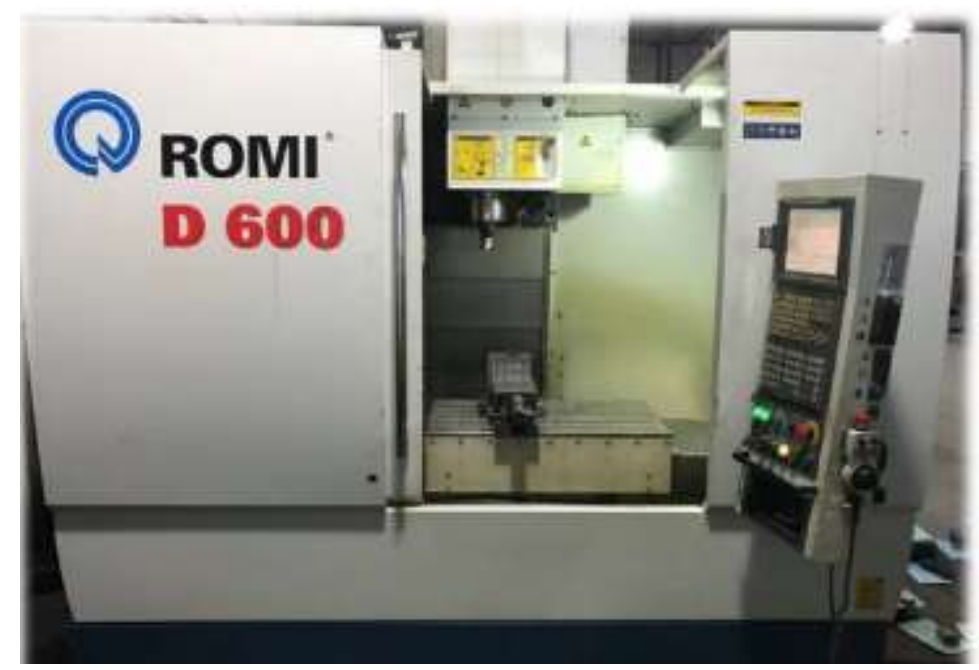

Fuente. Laboratorio CAD CAM- ESPOCH

Una de las facilidades para el desarrollo de este producto en cuestión, es la utilización de software CAD / CAM, el mismo que nos ayuda a determinar el diseño preliminar previo a la fabricación del molde mediante representación gráfica 3D, además de generar las estrategias de mecanizado del molde con su correspondiente simulación del mecanizado.[4]

En nuestro país la manufactura de accesorios en plastisol ha ido en aumento, aunque generalmente se lo realiza de una manera artesanal, teniendo como resultado la perdida de materia prima y tiempo principalmente. En este trabajo se establece las fases desarrolladas hasta llegar a tener un producto de en resina de buena calidad.[5]

Dentro de las interrogantes más importantes que se plantearon en el desarrollo de este proyecto fueron las siguientes:

¿Cuál es la proporción adecuada entre la base de plastisol y los colorantes?

¿Cuál es la temperatura adecuada de curado para el plastisol?

¿Cuál es el tiempo de permanencia del plastisol en el horno? 


\section{Metodología}

Las etapas que se desarrollan para llegar al producto final de esta investigación se muestran en la figura 2.

Figura. 2 Proceso CAD -CAM y producción del llavero en plastisol.

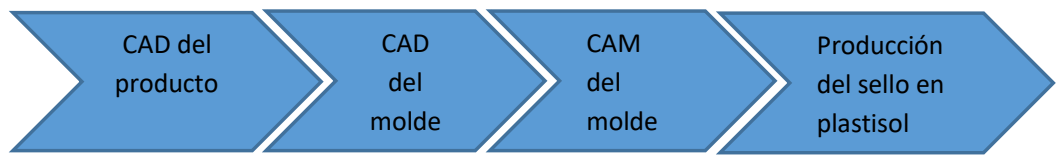

Los materiales que se utilizaron:

- $\quad$ Placa de aluminio 250x200x9 mm

- Bloque de grafito

- Fresas planas y buriles

- Base y pasta de colores de plastisol

\section{Resultados}

\section{Diseño asistido por computador (CAD)}

Como se muestra en la figura 3, se propone un modelado CAD del sello de la Escuela Superior Politécnica del Chimborazo (ESPOCH), el cual debe ser compacto, estético y bien elaborado. El diseño está en función de los requerimientos ergonómicos, estéticos y de acuerdo a la tecnología de mecanizado que disponemos en nuestro país, con el objetivo de obtener un producto estético, de calidad y precisión. [6]

Figura. 3 Diseño del sello de la ESPOCH .

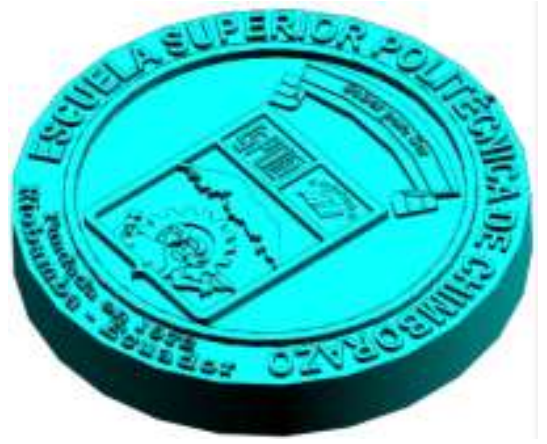

Durante el proceso de diseño hay que considerar el tamaño de los detalles del sello de tal manera que puedan ser mecanizados, considerando el diámetro de la herramienta a utilizar. En la figura 
4 se observa las posiciones de las cavidades que contiene la placa porta cavidades. (12 cavidades).

Figura. 4 Disposición de las cavidades del llavero ESPOCH

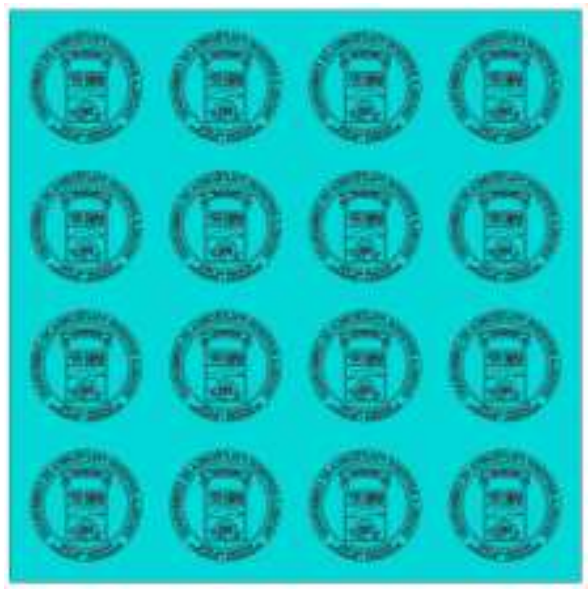

\section{Manufactura asistida por computador}

Luego de haber realizado los diseños mediante software CAD, se procedió a generar estrategias de mecanizado, para obtener código ISO G CNC, este archivo CNC con extensión .mpf permite trabajar con fresadora $\mathrm{CNC}$ con control Sinumerik. En la figura 5 se muestra una de las fases de mecanizado CNC del electrodo de grafito, por lo tanto se puede observar las líneas de movimiento de corte (trayectorias celeste) y las demás líneas corresponden a los movimientos en vacío, es decir sin corte.[7]

Figura. 5 CAM del electrodo

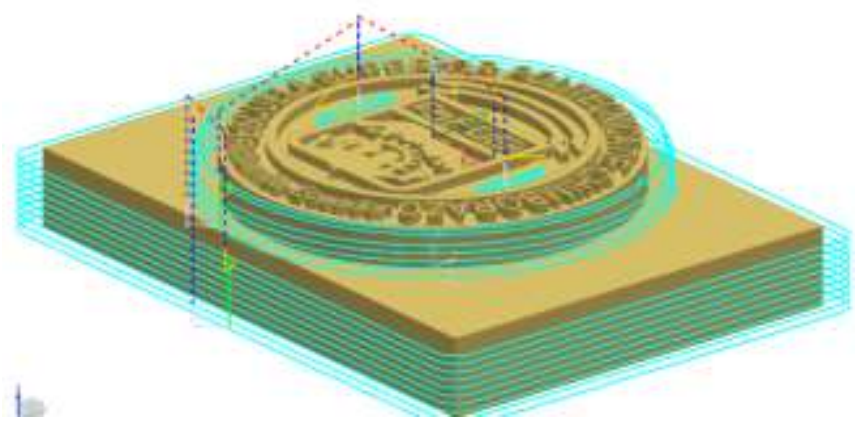

En la figura 6 se observa el proceso de mecanizado CNC del electrodo en grafito con medidas globales de 70x60x40mm, el mismo que tuvo una duración promedio de 6 horas, en el proceso mecanizado de los detalles del sello se utilizó un buril HSS de con 3mm de diámetro en el 
vástago, $0.4 \mathrm{~mm}$ en el lado de corte y un ángulo de punta de 8 grados, para el mecanizado de los contornos se utilizó una fresa plana de carburo de $6 \mathrm{~mm}$.

Figura. 6 Mecanizado del electrodo de grafito

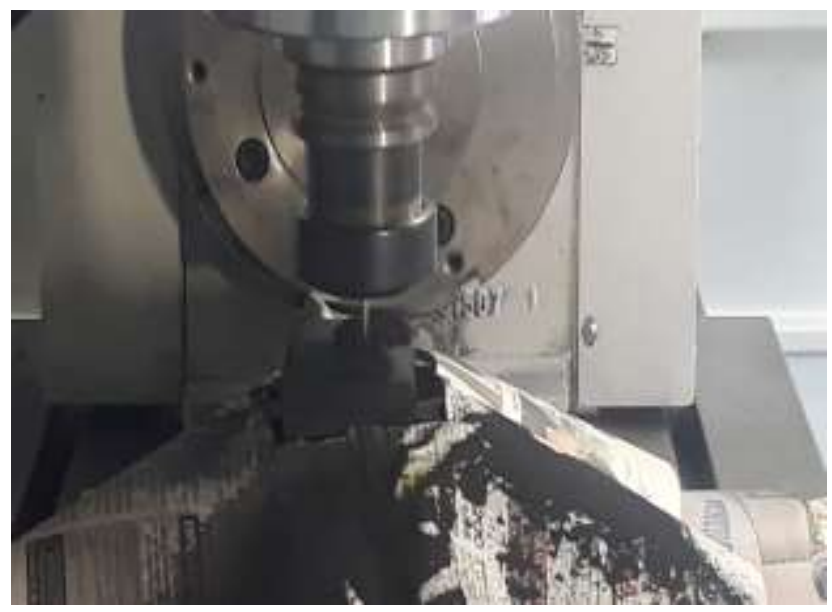

En la figura 7 se puede apreciar el mecanizado del electrodo del sello ESPOCH en función del diseño especificado en el software CAD, esta etapa es crucial ya que, de haber existido irregularidades o roturas en el mismo, el proceso se tenía que repetir hasta obtener un acabado de buena calidad.

\section{Figura. 7 Mecanizado el electrodo del sello de la ESPOCH}

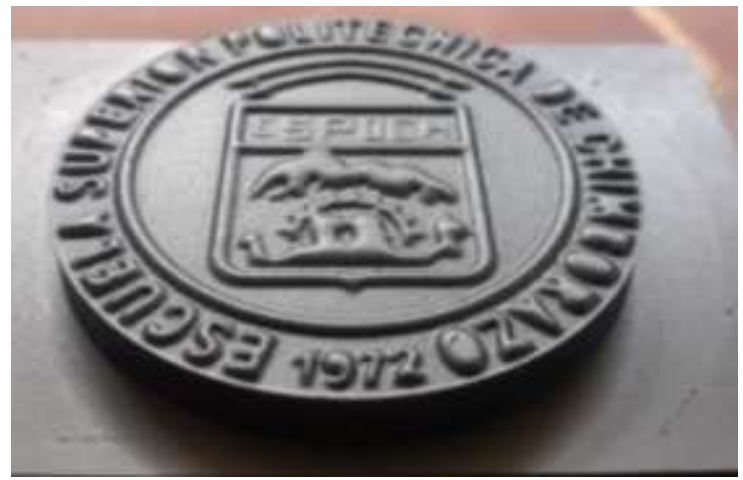

\section{Proceso de electroerosión de la placa porta cavidades.}

El siguiente proceso de mecanizado se denomina "electroerosión por penetración", que es un procedimiento de mecanizado que se basa en el hecho de arrancar material por medio de una serie sucesiva de descargar eléctricas, separadas unas de otras un cierto tiempo que saltan 
entre dos polos (electrodo y pieza a trabajar). Dichas descargar hacen que se calienten las piezas localmente a altas temperaturas $\left(30000\right.$ a $\left.50000{ }^{\circ} \mathrm{C}\right)$, así se van arrancando pequeños trozos del material en la pieza a trabajar quedando al final la superficie formada por pequeños cráteres que les dan un aspecto mate diferentes a los de mecanizado convencionales. [8] En la figura 8 se puede observar una máquina de electroerosión por penetración.

Figura. 8 Máquina de electroerosión por penetración

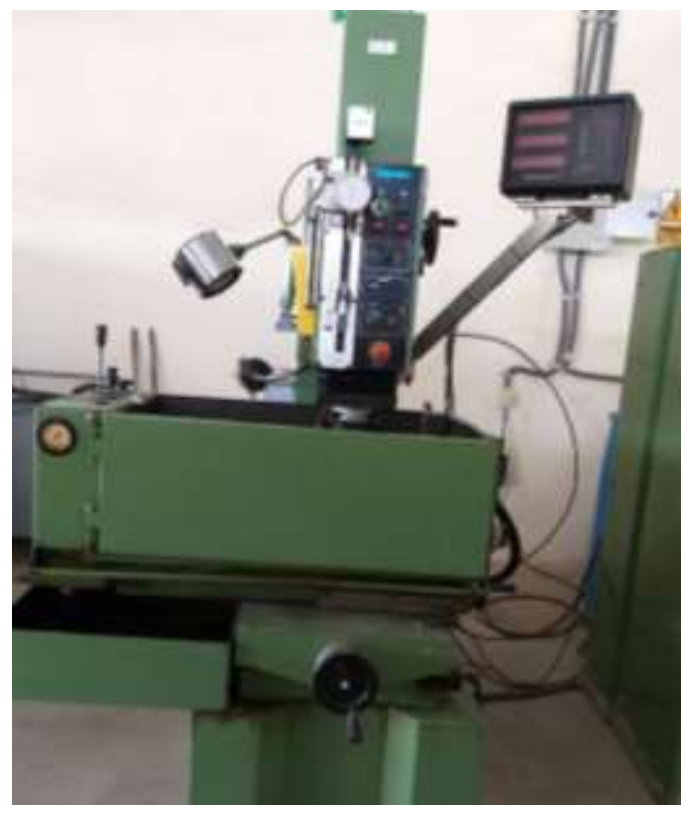

Fuente: Laboratorio CAD CAM -ESPOCH

En la figura 9 se puede apreciar el avance del proceso de electroerosión por penetración en una de las cavidades del molde, así como también la acción del dieléctrico (diésel) durante el proceso de limpieza del material carbonizado que se genera durante el proceso de erosión por descarga eléctrica.

El desgaste del electrodo fue notable, durante la vida útil del electrodo permite mecanizar 4 cavidades en desbaste y 8 cavidades en el proceso de afinado, en nuestro caso se mecanizó una profundidad de $0,8 \mathrm{~mm}$ por cavidad.

Figura. 9 Placa porta molde sello ESPOCH 


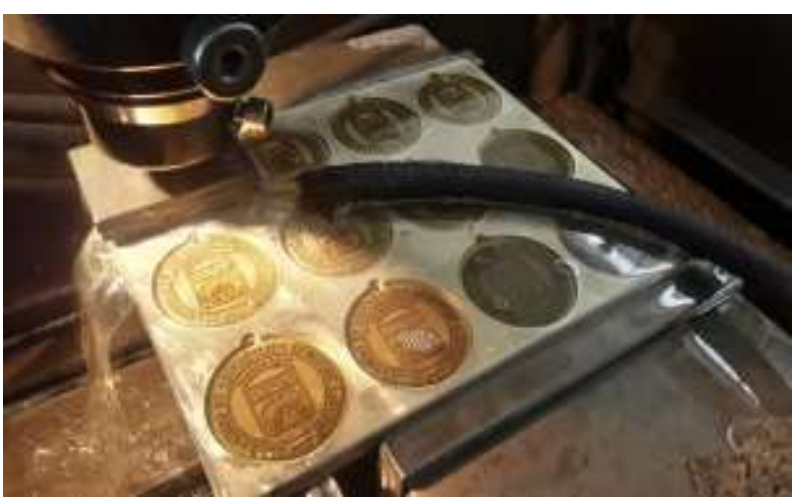

Luego de finalizar un trabajo de mecanizado por electroerosión de 2 horas por cavidad, se obtuvo la placa de 12 cavidades como se observa en la figura. 10

Figura. 10 Placa porta cavidades del sello ESPOCH

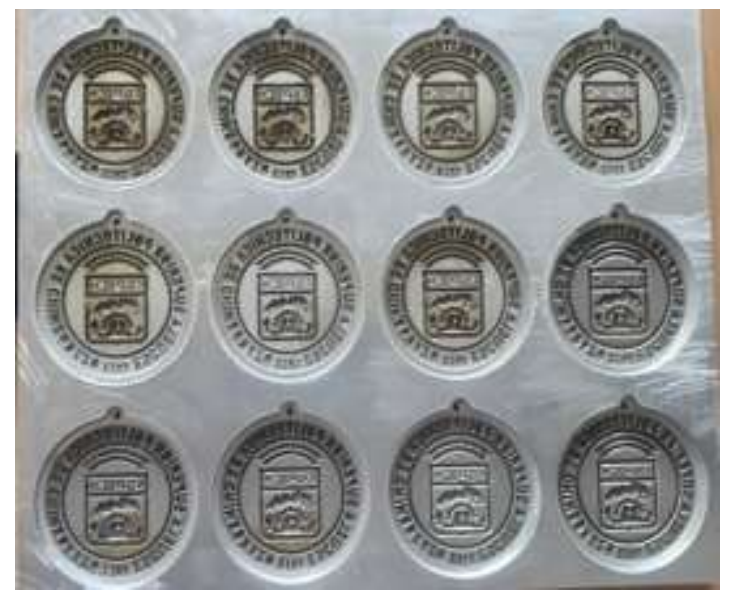

\section{Producción de los llaveros de plastisol}

Luego de obtener la placa mecanizada con el diseño del sello de la ESPOCH, entramos en la etapa de producción de los llaveros, en la cual los tiempos de curado, así como proporciones entre el plastisol y la base catalizadora son muy importantes, debido al hecho que tuvieron que ser analizadas y estudiadas con el fin de brindar un producto resistente y de buena calidad. [9]

Según la figura 11 se observa el sello de la ESPOCH, en la configuración del diseño original del sello se simplificaron algunos detalles, además se enfocó en los colores representativos del sello para personalizar el producto. Los aditivos de colores seleccionados para nuestro producto son: verde, rojo, dorado, blanco y negro, que lógicamente serán mezclados con la resina base de plastisol.

Figura. 11 Sello original de la ESPOCH 


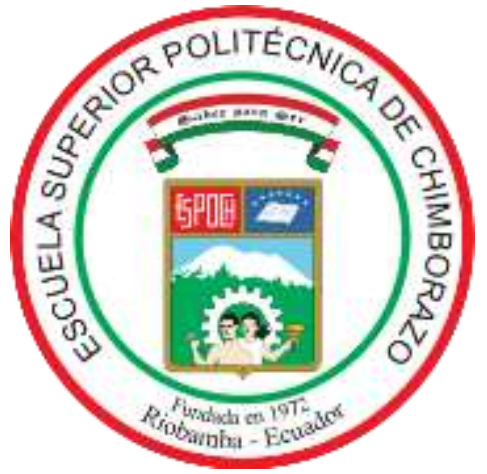

Durante la mezcla de la resina con los aditivos de color es muy importante añadir el catalizador en una proporción adecuada, para que durante el proceso de curado con temperatura del plastisol, se pueda obtener un producto flexible, resistente y con excelente acabado superficial.[10] Sin embargo, las proporciones de los materiales involucrados fueron la interrogante, por lo cual se realizó ensayos de prueba, con diferentes mezclas, con el objetivo de obtener una mezcla adecuada que nos permita obtener un producto idóneo.

Figura. 12. Resina plastisol y aditivos de color.

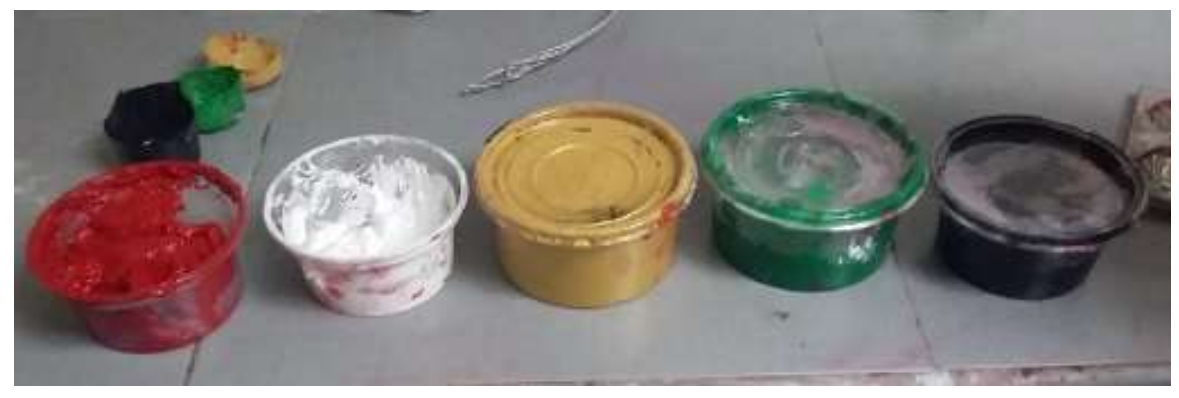

Para el proceso de curado por temperatura se utilizó un horno eléctrico con una temperatura máxima de $1300{ }^{\circ} \mathrm{C}$. En la producción del llavero en plastisol se empleó una temperatura de $300^{\circ}$ y con la puerta abierta.

Figura 19. Horno con resistencia eléctrica, $1300^{\circ} \mathrm{C}$

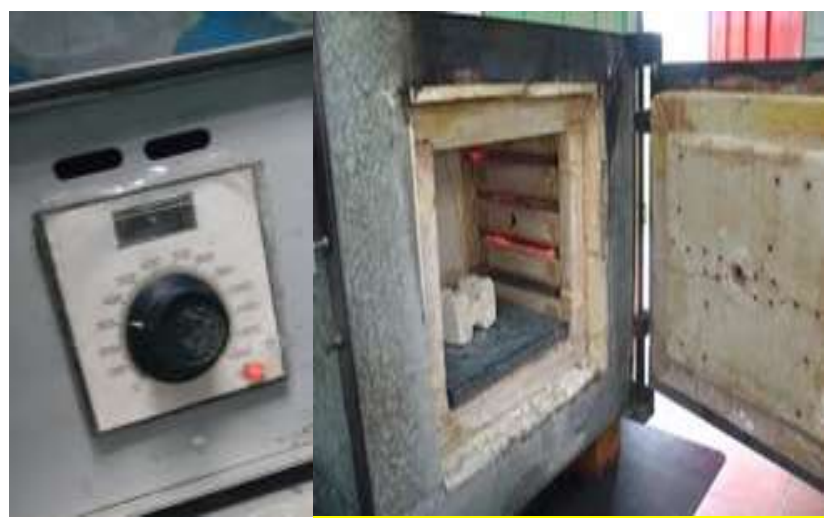


El proceso de obtención del sello en plastisol tiene las siguientes actividades:

1. Tener una mezcla con una proporción 3:1 (base:color), para cada uno de los colores hay que mezclar hasta obtener una solución homogénea.

2. Mediante jeringas número 10 se procede a recoger cada uno de los colores por separado.

3. Realizar la fase de inyección de los colores para cada uno de los detalles del sello (letras, símbolos y detalles del sello), es decir aquello que va a estar en alto relieve.

4. Se lleva el molde al horno a una temperatura de $300^{\circ} \mathrm{C}$ durante 1 minuto y 48 segundos con la puerta abierta del horno. Posteriormente se extrae el molde del horno. Ver figura 13.

5. Realizar el relleno de la cavidad del molde con resina de color blanco, a continuación, se vuelve a ingresar el molde al horno durante un tiempo de 4 minutos con la puerta abierta del horno.

6. Realizar la extracción del molde y posterior enfriamiento en agua.

En la figura 13, se observa el resultado posterior al proceso de curado de la resina que van a conformar los detalles del sello ESPOCH.

Figura. 13. Curado de los detalles del sello en plastisol.

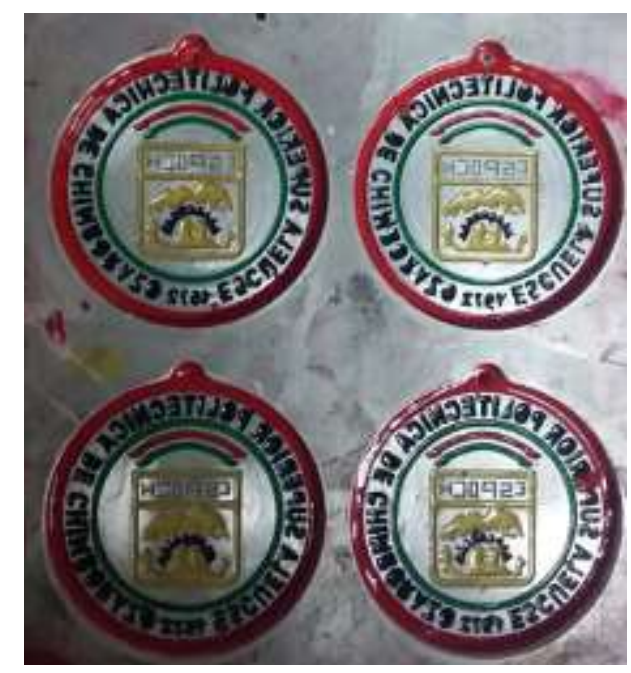

En la figura 14, se observa el relleno del sello con resina blanca y su reingreso al horno por segunda vez, para realizar el curado definitivo del producto.

Figura. 14. Curado del relleno del sello. 


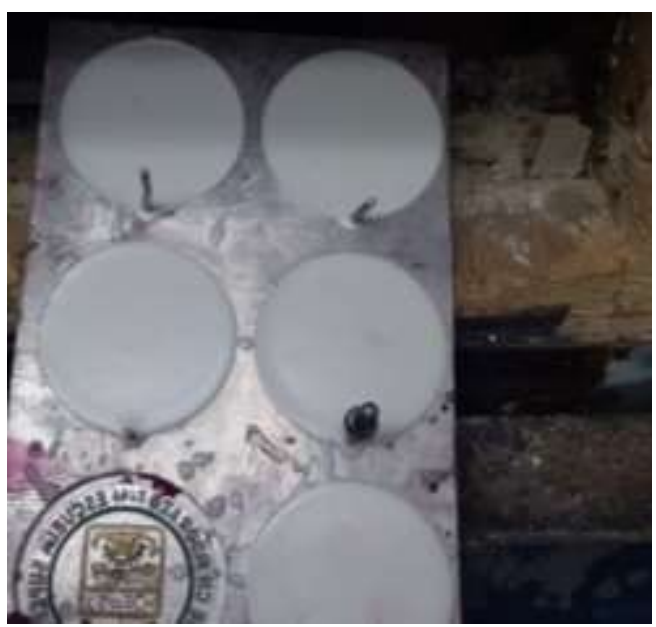

Luego de realizar el curado definitivo de los sellos en el horno, se procede a enfriar el molde en agua, el enfriamiento del molde ayuda al desmoldeo del producto. Finalmente se quitan las rebabas y se le adiciona una argolla al sello de plastisol. Ver figura 15.

Figura. 15. Sello en plastisol de la ESPOCH- producto terminado.

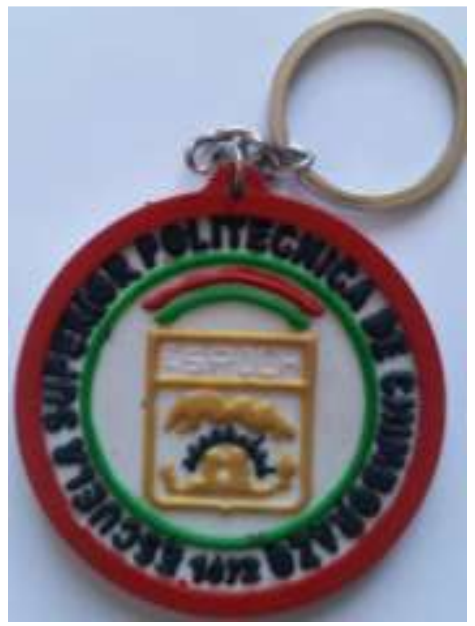

\section{Discusión}

\section{Experimentación del curado del plastisol.}

La proporción de las mezclas de la resina, el tiempo de curado y el tiempo de enfriamiento del molde son muy importantes para obtener un producto estético, resistente y de buena calidad. En las experimentaciones iniciales del curado de la resina, se obtuvo un producto que tiende a romperse, acabado superficial malo; esto como consecuencia de proporciones de mezclas incorrectas y falta de tiempo de curado. En la figura 16 se observa el proceso de curado en el horno con la puerta abierta. 
Figura. 16 Curado del plastisol por temperatura a $400^{\circ} \mathrm{C}$

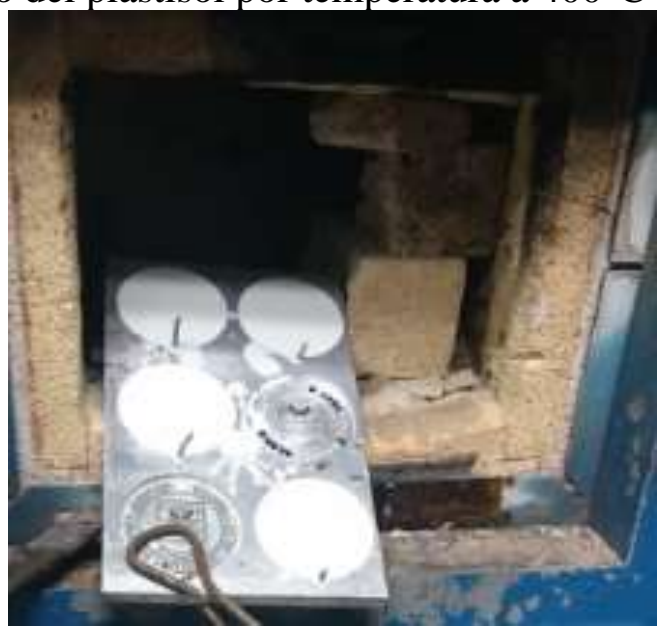

En la figura 17, se puede observar la presencia de porosidades por atrapamiento de gases, superficie externa quemada, sobre todo el defecto grave fue el agrietamiento del sello cuando se dobla el producto, la flexibilidad es importante para nuestro producto.

Figura. 17 Sello ESPOCH en plastisol - etapa de pruebas

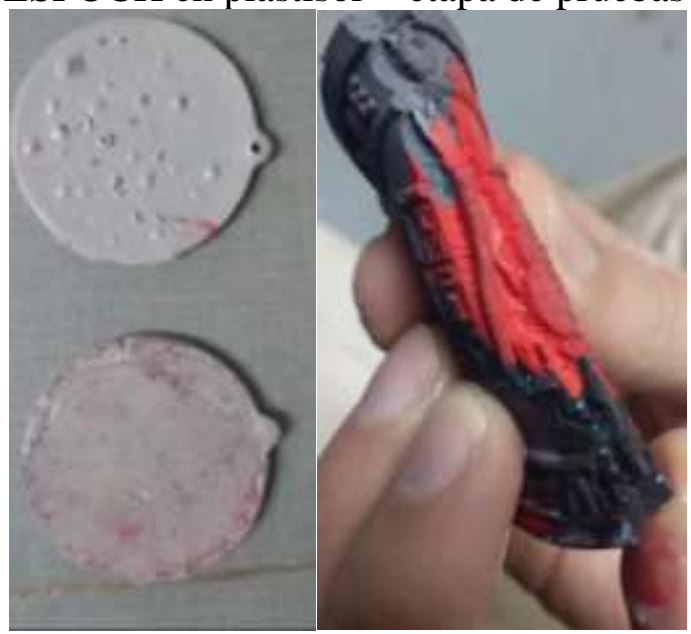

Una vez que se determinó las proporciones correctas de las mezclas de la resina, temperatura de curado, tiempos de curado, tiempo de enfriamiento del molde se procedió a producir los llaveros en serie.

\section{Resultados.}

Tanto las proporciones de mezcla, temperatura, los tiempos de curado y enfriamiento, son un factor importante para obtener productos de calidad y resistencia. A continuación, se resumen los resultados obtenidos durante la investigación:

Tabla 1 Parámetros de producción fallida del sello ESPOCH en plastisol. 


\begin{tabular}{|l|c|c|}
\hline \multicolumn{3}{|c|}{ Temperatura, tiempo de curado del plastisol } \\
\hline Color & Temperatura ${ }^{\circ} \mathrm{C}$ & $\begin{array}{c}\text { Tiempo } \\
\text { min/s }\end{array}$ \\
\hline Rojo & 200 & $1 \mathrm{~m} 0 \mathrm{~s}$ \\
\hline verde & 400 & $1 \mathrm{~m} 5 \mathrm{~s}$ \\
\hline Blanco & 100 & $2 \mathrm{~m} 10 \mathrm{~s}$ \\
\hline Negro & 100 & $1 \mathrm{~m} 55 \mathrm{~s}$ \\
\hline Dorado & 150 & $4 \mathrm{~m} 30 \mathrm{~s}$ \\
\hline \multicolumn{3}{|c|}{ Proporción de mezcla } \\
\hline \multicolumn{1}{|c|}{ Color } & $\begin{array}{c}\text { Proporción } \\
\text { base (\%) }\end{array}$ & $\begin{array}{c}\text { Proporción } \\
\text { color (\%) }\end{array}$ \\
\hline Rojo & 60 & 40 \\
\hline Verde & 60 & 40 \\
\hline Blanco & 60 & 40 \\
\hline Negro & 60 & 40 \\
\hline Dorado & 60 & 40 \\
\hline
\end{tabular}

Tabla 2 Parámetros de producción del sello ESPOCH en plastisol.

\begin{tabular}{|c|c|c|}
\hline \multicolumn{3}{|c|}{ Temperatura, tiempo de curado del plastisol. } \\
\hline Color & $\begin{array}{c}\text { Temperatura } \\
{ }^{\circ} \mathrm{C}\end{array}$ & $\begin{array}{l}\text { Tiempo } \\
(\mathrm{min} / \mathrm{s})\end{array}$ \\
\hline Rojo & 300 a 400 & $0 \mathrm{~h} / 1 \mathrm{~m} / 48 \mathrm{~s}$ \\
\hline Verde & 300 a 400 & $1 \mathrm{~m} / 48 \mathrm{~s}$ \\
\hline Blanco & 300 a 400 & $4 \mathrm{~m} / 0 \mathrm{~s}$ \\
\hline Negro & 300 a 400 & $1 \mathrm{~m} / 48 \mathrm{~s}$ \\
\hline Dorado & 300 a 400 & $1 \mathrm{~m} / 48 \mathrm{~s}$ \\
\hline \multicolumn{3}{|c|}{$\begin{array}{l}\text { Proporción de mezcla del plastisol } \\
\end{array}$} \\
\hline Color & $\begin{array}{c}\text { Proporción de } \\
\text { base. }(\%)\end{array}$ & $\begin{array}{l}\text { Proporción } \\
\text { del color }(\%)\end{array}$ \\
\hline Rojo & 75 & 25 \\
\hline Verde & 75 & 25 \\
\hline Blanco & 80 & 20 \\
\hline Negro & 75 & 25 \\
\hline Dorado & 75 & 25 \\
\hline
\end{tabular}

\section{Conclusiones.}

- Se debe determinar apropiadamente las dimensiones geométricas de cada uno de los detalles que contiene el sello, de tal manera que la tecnología de mecanizado CNC permita obtener un electrodo en grafito con buena definición.

- Para obtener la mezcla adecuada de resina para la producción del sello de la ESPOCH, se determinó que, para los colores rojo, negro y morado, la mezcla que mejor resultado ofrece es un $75 \%$ de resina base y un $25 \%$ de aditivo para dar el color. En el caso de la mezcla de color blanco se determina una proporción de $80 \%$ de resina base y un $20 \%$ de aditivo para dar el color.

- La temperatura promedio de curado para la producción del llavero en plastisol es de $300^{\circ} \mathrm{C}$ y con la puerta del horno abierta. Con esta temperatura se obtuvo mejores resultados en cuanto a calidad y resistencia del producto. 
- El tiempo de curado del plastisol depende principalmente del espesor de la capa de resina, para nuestro caso el curado se lo realizo en dos etapas: la primera fue curar las resinas de colores que van a conformar los detalles del sello, en un tiempo promedio de $1 \mathrm{~min}$ con $48 \mathrm{~S}$; finalmente se curó relleno del llavero que tiene mayor cantidad de resina, por lo que lógicamente se incrementó el tiempo, siendo un tiempo promedio de 4 min aproximadamente. Estos parámetros se complementan con la temperatura del horno de $300^{\circ} \mathrm{C}$ y con la puerta del horno abierta.

- La omisión de los parámetros recomendados para la obtención de productos en plastisol, ocasiona que el producto se queme, porosidad, acabado superficial deficiente, resistencia mecánica baja, entre otros.

\section{Referencias Bibliográficas.}

[1] S. Kalpakjian and S. Schmid, "Manufacturing Processes," Pearson, 2008.

[2] Richard A Gizelbach, CNC Machining. 2018.

[3] W. F. Smith, J. Hashemi, G. N. Cázares, and P. A. González-Caver, Fundamentos de la ciencia e ingenier $\backslash \backslash$ i\}a de materiales. McGraw-Hill México, DF, 2006.

[4] J. J. Shah and M. Mäntylä, Parametric and feature-based CAD/CAM: concepts, techniques, and applications. John Wiley \& Sons, 1995.

[5] H. A. T. MONSIVAIS, "Estudio de factores que afectan la calidad de proceso y producto a base de plastisol," 2018.

[6] M. F. Ashby and D. Cebon, "Materials selection in mechanical design," Le J. Phys. $I V$, vol. 3, no. C7, pp. C7--1, 1993.

[7] W. D. Callister, D. G. Rethwisch, P. Molera Solà, and N. S. Ballesteros, Ciencia e ingenier $\bigwedge 1$ i\}a de materiales, vol. 1. Reverté, 2016.

[8] C. J. Santamarl'lia Zambrano, "Análisis de parámetros de mecanizado en el proceso de electroerosión por penetración en microfundición de aluminio con electrodos de cobre y grafito y su relación con la rugosidad superficial resultante," Universidad Técnica de Ambato. Facultad de Ingenier $\{\backslash ’ \backslash i\}$ a Civil y Mecánica ..., 2017.

[9] M. I. Beltrán and others, "Tema 2. Tipos de plásticos, aditivación y mezclado," Tecnol. los Pol^ \ijmeros, 2011.

[10] J. F. Shackelford, Ciencia de materiales para ingenieros. Prentice Hall Hispanoamericana, 1995. 


\section{PARA CITAR EL ARTÍCULO INDEXADO.}

Escobar Guachambala, M., Aquino Arroba, S., Pozo Safla, E., \& Choto Chariguaman, L. (2019). Determinación de los parámetros de diseño, manufactura y producción de sellos en plástico. Ciencia Digital, 3(3.1), 188-202. https://doi.org/10.33262/cienciadigital.v3i3.1.688

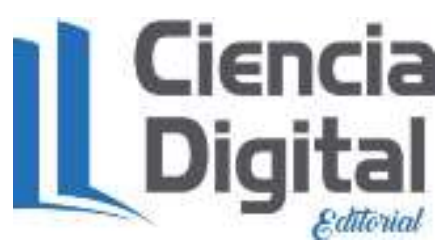

El artículo que se publica es de exclusiva responsabilidad de los autores y no necesariamente reflejan el pensamiento de la Revista Ciencia Digital.

El artículo queda en propiedad de la revista y, por tanto, su publicación parcial y/o total en otro medio tiene que ser autorizado por el director de la Revista Ciencia Digital.
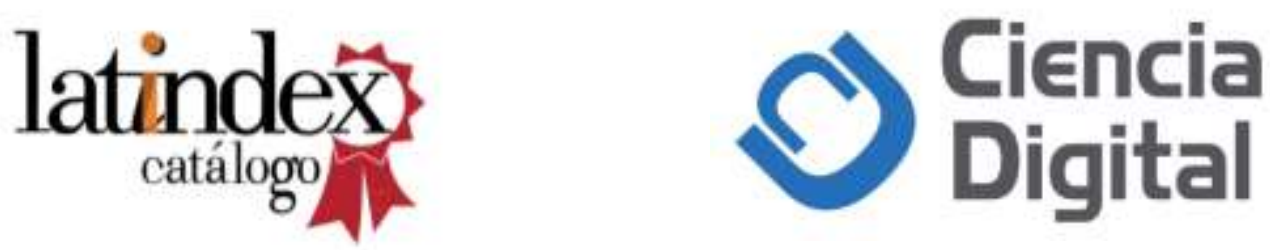